\title{
Master-slave approach for the modelling of joints with dependent degrees of freedom in flexible mechanisms
}

\author{
J. Muñoz*, G. Jelenić and M. A. Crisfield \\ Department of Aeronautics, Imperial College of Science, Technology and Medicine, London, UK
}

\begin{abstract}
SUMMARY
The analysis of multibody systems requires an exact description of the kinematics of the joints involved. In the present work the master-slave approach is employed and endowed with the possibility of including several more complex types of joints. We present the formulation for joints where some relation between the different released degrees of freedom exists such as the screw joint, the rack-and-pinion joint or the cam joint. These joints are implemented in conjunction with geometrically exact beams and an energy-momentum conserving time-stepping algorithm.
\end{abstract}

KEY WORDS: multibody dynamics; flexible mechanisms; conserving time-integration; master-slave

technique, constraint violation

\section{INTRODUCTION}

In this paper we extend a method for dynamic analysis of flexible mechanisms [1-3] to more complex joints including screw joint, rack-and-pinion joint and cam joint. The essential ingredients of the method are the geometrically exact treatment of the beam deformation in presence of large displacements and rotations [4-8] and exact non linear kinematics of joints using the minimum set of parameters $[2,3,9]$.

Several techniques can be found in the literature of which the method of Lagrangian multipliers, the penalty method and the augmented Lagrangian method are widely used [10,11]. The present work is motivated by the authors' earlier work on geometrically non-linear static and dynamic formulations for 3D beams with end releases, whereby the non-linear 3D kinematics of the joints was handled using the so-called master-slave technique [1-3], also known as the minimum set method. Further results in this area have been given in Reference [9]. In the master-slave approach, it is the relationship between the variations of the nodal positions in a spatially discretised weak form of the problem that defines the character of the joint in

\footnotetext{
*Correspondence to: J. Muñoz, Department of Aeronautics, Imperial College, Prince Consort Road, London SW7 2BY, U.K.

Deceased.

Contract/grant sponsor: Engineering and Physical Sciences Research Council; contract/grant number: GR/R 04171/01 and AF/100089.
} 
question. In this sense, the approach seems to be particularly convenient for finite-element implementation, whereby the compatibility relationships of this type are handled at the point of assembling the structural (linearised) equilibrium from the linearized element equilibria. The kinematics of the joints is therefore treated directly, without resorting to Lagrangian multipliers or penalty stiffnesses, thus resulting in a procedure with a minimal number of parameters.

In Section 2 the general master-slave formulation is introduced and embedded in the finite element context and in Section 3 its modification in order to allow more complex joints with some dependence between the released degrees of freedom is presented where their applications and advantages are highlighted. Section 4 introduces an energy and momentum conserving algorithm that is adapted to joints with dependent degrees of freedom. Two numerical examples are presented in Section 5.

\section{MASTER-SLAVE APPROACH}

The master-slave approach has been described in detail in References [2,3] and will be summarized here in order to prepare the ground for the modifications needed for the implementation of the more complex joints. A joint will be formed when two elements of a system are not rigidly attached to each other. The kinematic relationship between two nodes of two different element ends connected to the same joint can be given as an algebraic equation. In the master-slave approach the degrees of freedom (dof) of one of the two nodes (the slave node) are referred to the degrees of freedom of the other node (the master node) through the released degrees of freedom (relative displacement and rotation of the slave node with respect to the master node given in the body attached frame) denoted by $\boldsymbol{p}_{\mathrm{R}}=\left\langle\boldsymbol{r}_{\mathrm{R}}^{\mathrm{T}} \boldsymbol{\theta}_{\mathrm{R}}^{\mathrm{T}}\right\rangle^{\mathrm{T}}$, whereas $\boldsymbol{p}_{\mathrm{m}}=\left\langle\boldsymbol{r}_{\mathrm{m}}^{\mathrm{T}} \boldsymbol{\theta}_{\mathrm{m}}^{\mathrm{T}}\right\rangle^{\mathrm{T}}$ and $\boldsymbol{p}_{\mathrm{s}}=\left\langle\boldsymbol{r}_{\mathrm{s}}^{\mathrm{T}} \boldsymbol{\theta}_{\mathrm{s}}^{\mathrm{T}}\right\rangle^{\mathrm{T}}$ are the displacements and rotations of the master and slave nodes given in the inertial frame (see Figure 1 where the nomenclature is indicated).

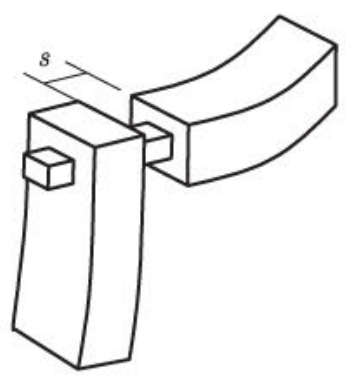

(a)

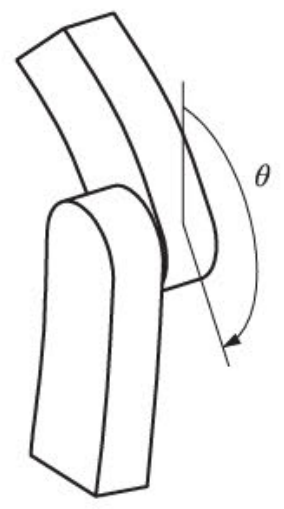

(b)

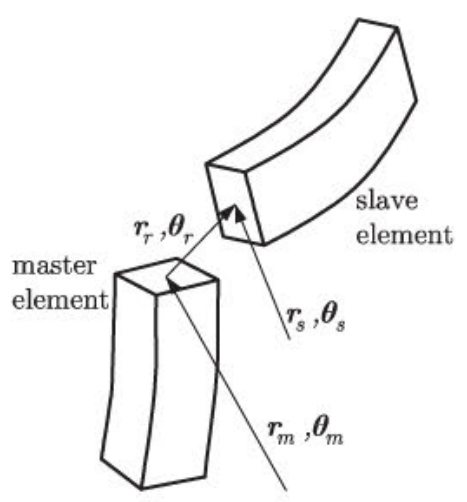

(c)

Figure 1. (a) Prismatic joint; (b) revolute joint; and (c) notation used. 
The kinematic relation between the master and slave dof may be written as follows:

$$
\begin{aligned}
\boldsymbol{r}_{\mathrm{s}} & =\boldsymbol{r}_{\mathrm{m}}+\boldsymbol{r}_{\mathrm{r}}=\boldsymbol{r}_{\mathrm{m}}+\boldsymbol{\Lambda}_{\mathrm{m}} \boldsymbol{r}_{\mathrm{R}} \\
\boldsymbol{\Lambda}_{\mathrm{s}} & =\boldsymbol{\Lambda}_{\mathrm{m}} \boldsymbol{\Lambda}_{\mathrm{R}}=\boldsymbol{\Lambda}_{\mathrm{r}} \boldsymbol{\Lambda}_{\mathrm{m}}
\end{aligned}
$$

where $\boldsymbol{r}_{\mathrm{r}}=\boldsymbol{\Lambda}_{\mathrm{m}} \boldsymbol{r}_{\mathrm{R}}$ and $\boldsymbol{\Lambda}_{\mathrm{r}}=\boldsymbol{\Lambda}_{\mathrm{m}} \boldsymbol{\Lambda}_{\mathrm{R}} \boldsymbol{\Lambda}_{\mathrm{m}}^{\mathrm{T}}$ are the released translations and the triad of released rotations referred to the inertial frame, with $\boldsymbol{\Lambda}_{\mathrm{s}}=\exp \left[\hat{\boldsymbol{\theta}}_{\mathrm{s}}\right], \boldsymbol{\Lambda}_{\mathrm{m}}=\exp \left[\hat{\boldsymbol{\theta}}_{\mathrm{m}}\right]$ and $\boldsymbol{\Lambda}_{\mathrm{R}}=\exp \left[\hat{\boldsymbol{\theta}}_{\mathrm{R}}\right]$ being the orthogonal tensors in $\mathbb{R}^{3}$. Note that $\boldsymbol{r}_{\mathrm{R}}$ and $\boldsymbol{\Lambda}_{\mathrm{R}}$ are the released translations and the triad of released rotations referred to the body attached frame. Here and throughout the paper a hat $(\widehat{)})$ will be used to denote the skew-symmetric matrix of a $3 \mathrm{D}$ vector $\boldsymbol{v}$ such that $\hat{\boldsymbol{v}} \boldsymbol{w}=\boldsymbol{v} \times \boldsymbol{w}=-\boldsymbol{w} \times \boldsymbol{v}=-\hat{\boldsymbol{w}} \boldsymbol{v}=\hat{\boldsymbol{w}}^{\mathrm{T}} \boldsymbol{v}$ for any $3 \mathrm{D}$ vector $\boldsymbol{w}$. It is convenient to work with the incremental rotation, denoted as $\omega_{\mathrm{R}}$, which measures the relative rotation between time steps, i.e.

$$
\boldsymbol{\Lambda}_{\mathrm{R}, n+1}=\exp \left[\hat{\boldsymbol{\omega}}_{\mathrm{R}}\right] \boldsymbol{\Lambda}_{\mathrm{R}, n}
$$

where here and throughout the paper indices $n$ and $n+1$ will denote the value of a quantity at times $t_{n}$ and $t_{n+1}$. Kinematic equations (1) may be varied to obtain $[2,3]$

$$
\begin{aligned}
\delta \boldsymbol{r}_{\mathrm{s}} & =\delta \boldsymbol{r}_{\mathrm{m}}-\widehat{\boldsymbol{\Lambda}_{\mathrm{m}}} \boldsymbol{r}_{\mathrm{R}} \delta \boldsymbol{\vartheta}_{\mathrm{m}}+\boldsymbol{\Lambda}_{\mathrm{m}} \delta \boldsymbol{r}_{\mathrm{R}} \\
\delta \boldsymbol{\vartheta}_{\mathrm{s}} & =\delta \boldsymbol{\vartheta}_{\mathrm{m}}+\boldsymbol{\Lambda}_{\mathrm{m}} \mathbf{H}\left(\boldsymbol{\omega}_{\mathrm{R}}\right) \delta \boldsymbol{\omega}_{\mathrm{R}}
\end{aligned}
$$

where $\delta \boldsymbol{\vartheta}_{\mathrm{s}}$ and $\delta \boldsymbol{\vartheta}_{\mathrm{m}}$ are the spin rotational variations of $\boldsymbol{\Lambda}_{\mathrm{s}}$ and $\boldsymbol{\Lambda}_{\mathrm{m}}$, obtained from $\delta \boldsymbol{\Lambda}_{\mathrm{s}}=\widehat{\delta \boldsymbol{\vartheta}_{\mathrm{s}}} \boldsymbol{\Lambda}_{\mathrm{s}}$ and $\delta \boldsymbol{\Lambda}_{\mathrm{m}}={\widehat{\delta \boldsymbol{\vartheta}_{\mathrm{m}}}}_{\boldsymbol{\Lambda}_{\mathrm{m}}}$ and matrix $\mathbf{H}\left(\boldsymbol{\omega}_{\mathrm{R}}\right)$ is defined as $[4,5,8]$

$$
\mathbf{H}\left(\omega_{\mathrm{R}}\right)=\mathbf{I}+\frac{1-\cos \left|\omega_{\mathrm{R}}\right|}{\left|\omega_{\mathrm{R}}\right|^{2}} \hat{\omega}_{\mathrm{R}}+\frac{1}{\left|\omega_{\mathrm{R}}\right|^{2}}\left(1-\frac{\sin \left|\omega_{\mathrm{R}}\right|}{\left|\omega_{\mathrm{R}}\right|}\right) \hat{\omega}_{\mathrm{R}}^{2}
$$

Equations (2) and (3) can be written in a compact form as

$$
\delta \boldsymbol{p}_{\mathrm{s}}=\mathbf{N} \delta \boldsymbol{p}_{\mathrm{Rm}}
$$

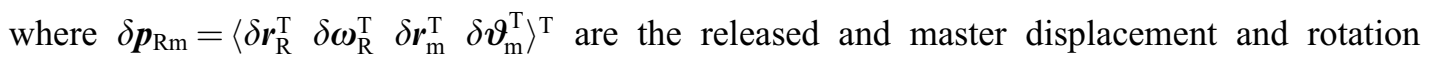
variations and $\delta \boldsymbol{p}_{\mathrm{s}}=\left\langle\delta \boldsymbol{r}_{\mathrm{s}}^{\mathrm{T}} \delta \boldsymbol{\vartheta}_{\mathrm{s}}^{\mathrm{T}}\right\rangle^{\mathrm{T}}$ are the slave displacement and rotation variations. The matrix $\mathbf{N}$ has the following expression

$$
\mathbf{N}=\left[\begin{array}{cccc}
\boldsymbol{\Lambda}_{\mathrm{m}} & \mathbf{0} & \mathbf{I} & -\widehat{\boldsymbol{\Lambda}_{\mathrm{m}} \boldsymbol{r}_{\mathrm{R}}} \\
\mathbf{0} & \boldsymbol{\Lambda}_{\mathrm{m}} \mathbf{H}\left(\omega_{\mathrm{R}}\right) & \mathbf{0} & \mathbf{I}
\end{array}\right]
$$

The general finite element formulation of a beam element with $N$ nodes leads to the following discretised weak form $[6,8]$

$$
G=\sum_{i=1}^{N} \delta \boldsymbol{p}_{\mathrm{s}}^{i} \cdot \boldsymbol{g}^{i}=0
$$

where $\boldsymbol{g}^{i}=\left\langle\boldsymbol{g}_{f}^{i \mathrm{~T}} \boldsymbol{g}_{\phi}^{i \mathrm{~T}}\right\rangle^{\mathrm{T}}$ is the dynamic residual including the internal, inertial and external load vectors of node $i$ and $\boldsymbol{g}_{f}^{i}$ and $\boldsymbol{g}_{\phi}^{i}$ correspond to its force and moment parts. Using the matrix 
$\mathbf{N}$ in (6) and replacing (5) into (7) gives rise to

$$
\sum_{i=1}^{N} \delta \boldsymbol{p}_{\mathrm{Rm}}^{i} \cdot \mathbf{N}_{i}^{\mathrm{T}} \boldsymbol{g}^{i}=\mathbf{0}
$$

The arbitrariness of the virtual displacements leads to the following system of equations

$$
\mathbf{N}_{i}^{\mathrm{T}} \boldsymbol{g}^{i}=\overline{\boldsymbol{g}}^{i}=\mathbf{0}
$$

The details about the linearisation procedure and the solution process of the non-linear equations (8) will not be described here but can be found in References [2,12].

\section{JOINTS WITH DEPENDENT RELEASED DOF}

In this section the above results will be modified in order to model joints where some of the released degrees of freedom are dependent on some independent released dof. Three examples are shown in Figure 2: the cam joint, the rack-and-pinion joint and the screw joint. Other joints such as worm gears, helical gears or bevel gears [13] also fall into this category. In general, the relation between the two dependent degrees of freedom is non-linear. A general setting will be presented first and will be applied later for the screw, rack-and-pinion and cam joints.

\subsection{General case}

Setting the released displacement $\boldsymbol{r}_{\mathrm{R}}$ as the dependent variable and the released rotation $\boldsymbol{\theta}_{\mathrm{R}}$ as the independent variable, the relationship between the two of them may be written through a function $\boldsymbol{f} \in C^{2}: \mathbb{R}^{3} \rightarrow \mathbb{R}^{3}$,

$$
\boldsymbol{r}_{\mathrm{R}}=\boldsymbol{f}\left(\boldsymbol{\theta}_{\mathrm{R}}\right)
$$

In the joints commonly considered, only one component of the released rotation will be different from zero, in which case $\boldsymbol{\theta}_{\mathrm{R}}$ and $\omega_{\mathrm{R}}$ (total and incremental released rotations)

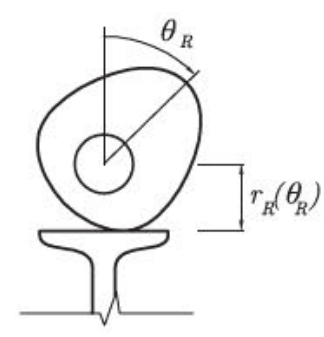

(a)

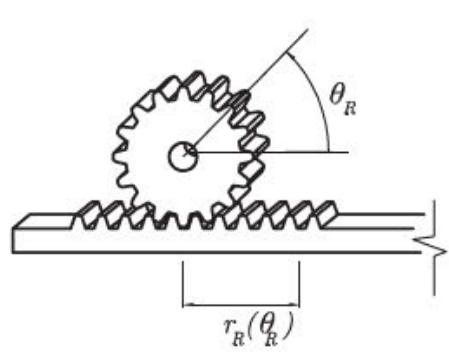

(b)

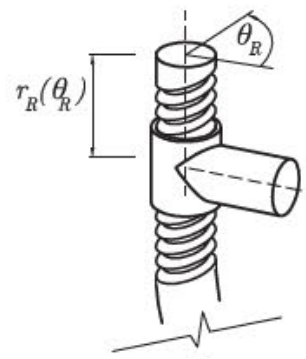

(c)

Figure 2. Examples of complex joints with dependent released degrees of freedom: (a) cam joint; (b) rack-and-pinion joint; and (c) screw joint. 


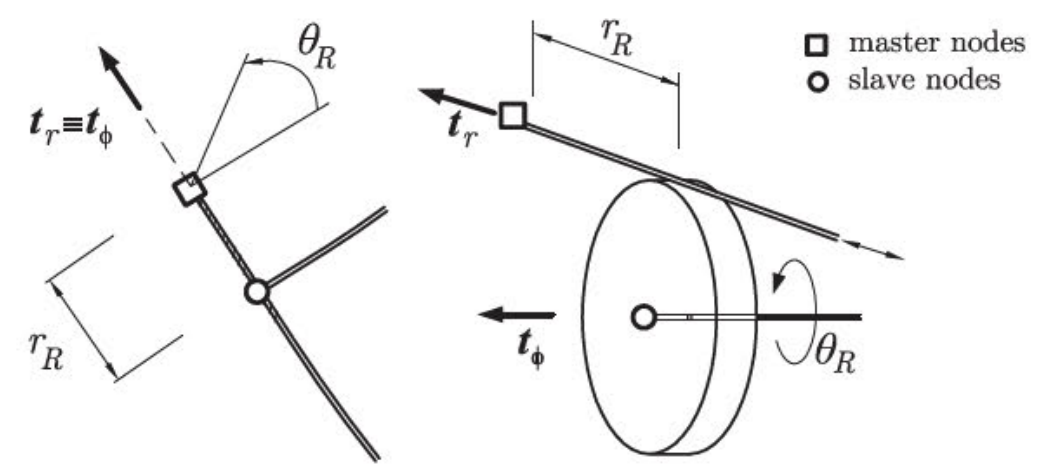

Figure 3. Scheme of the screw joint and the rack-and-pinion joint.
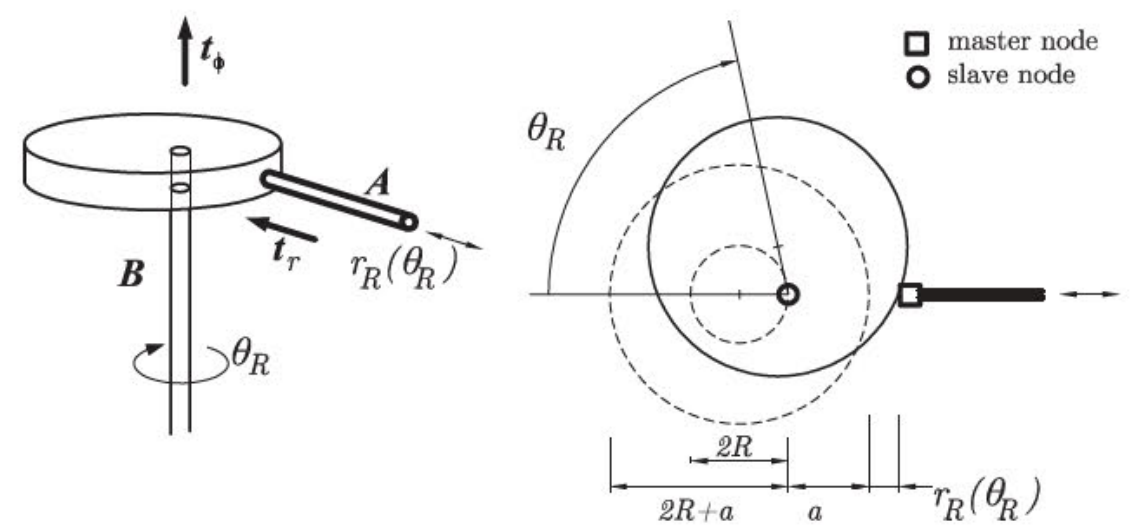

Figure 4. Scheme of the cam joint.

are in the same direction. Therefore $\delta \boldsymbol{\theta}_{\mathrm{R}}=\delta \omega_{\mathrm{R}}$, and the variation of (9) reads

$$
\delta \boldsymbol{r}_{\mathrm{R}}=\frac{\partial \boldsymbol{f}\left(\boldsymbol{\theta}_{\mathrm{R}}\right)}{\partial \boldsymbol{\theta}_{\mathrm{R}}} \delta \omega_{\mathrm{R}}
$$

where $\partial \boldsymbol{f}\left(\boldsymbol{\theta}_{\mathrm{R}}\right) / \partial \boldsymbol{\theta}_{\mathrm{R}}$ is a matrix of partial derivatives of the components of $\boldsymbol{f}\left(\boldsymbol{\theta}_{\mathrm{R}}\right)$ with respect to the components of $\boldsymbol{\theta}_{\mathrm{R}}$. Replacing $\delta \boldsymbol{r}_{\mathrm{R}}$ into (2) turns the matrix $\mathbf{N}$ into

$$
\underline{\mathbf{N}}=\left[\begin{array}{cccc}
\mathbf{0} & \boldsymbol{\Lambda}_{\mathrm{m}} \frac{\partial \boldsymbol{f}\left(\boldsymbol{\theta}_{\mathrm{R}}\right)}{\partial \boldsymbol{\theta}_{\mathrm{R}}} & \mathbf{I} & -\widehat{\boldsymbol{\Lambda}_{\mathrm{m}} \boldsymbol{r}_{\mathrm{R}}} \\
\mathbf{0} & \boldsymbol{\Lambda}_{\mathrm{m}} \mathbf{H}\left(\omega_{\mathrm{R}}\right) & \mathbf{0} & \mathbf{I}
\end{array}\right]
$$

The three joints in Figure 2 have important common features that allow us to study them from a single standpoint. In all cases there exists a released rotation around axis $\boldsymbol{t}_{\phi}$ (see Figures 3 and 4), and a released displacement with components along axes $t_{\mathrm{r}}$ (attached to the master node) and $\boldsymbol{t}_{\mathrm{r}} \times \boldsymbol{t}_{\phi}$. 


\subsection{Screw joint and rack-and-pinion joint}

For the screw joint axes $\boldsymbol{t}_{\mathrm{r}}$ and $\boldsymbol{t}_{\phi}$ are coincident, while in the rack-and-pinion joint they are perpendicular to each other (see Figure 3 ). The latter also has a non-zero initial displacement (the radius of the pinion) in a direction perpendicular to $\boldsymbol{t}_{\mathrm{r}}$ and $\boldsymbol{t}_{\phi}$.

The particular form of (9) for the rack-and-pinion joint (rp) and the screw joint (sc) can be written using the scalar parameters $b$ and $c$ as

$$
\boldsymbol{r}_{\mathrm{R}}=\boldsymbol{f}_{\mathrm{r} p}\left(\boldsymbol{\theta}_{\mathrm{R}}\right)=\boldsymbol{f}_{\mathrm{sc}}\left(\boldsymbol{\theta}_{\mathrm{R}}\right)=b\left(\boldsymbol{t}_{\mathrm{r}} \times \boldsymbol{t}_{\phi}\right)+c\left(\boldsymbol{\theta}_{\mathrm{R}} \cdot \boldsymbol{t}_{\phi}\right) \boldsymbol{t}_{\mathrm{r}}=b \hat{\boldsymbol{t}}_{\mathrm{r}} \boldsymbol{t}_{\phi}+c\left(\boldsymbol{t}_{\mathrm{r}} \otimes \boldsymbol{t}_{\phi}\right) \boldsymbol{\theta}_{\mathrm{R}}
$$

where $b$ is the radius of the pinion and $c$ has a different meaning for the two joints. In the rack-and-pinion joint $c=b$ and in the screw joint it corresponds to the pitch of the thread. Note that since $\boldsymbol{t}_{\mathrm{r}}$ and $\boldsymbol{t}_{\phi}$ have the same direction in the screw joint, the constant term $b \hat{\boldsymbol{t}}_{\mathrm{r}} \boldsymbol{t}_{\phi}$ in this case vanishes. Applying the directional derivative to the kinematic relation (12), equation (10) gives rise to the term $\partial \boldsymbol{f}\left(\boldsymbol{\theta}_{\mathrm{R}}\right) / \partial \boldsymbol{\theta}_{\mathrm{R}}$, needed in (11), for both joints as

$$
\left.\frac{\partial \boldsymbol{f}\left(\boldsymbol{\theta}_{\mathrm{R}}\right)}{\partial \boldsymbol{\theta}_{\mathrm{R}}}\right|_{\mathrm{r} p}=\left.\frac{\partial \boldsymbol{f}\left(\boldsymbol{\theta}_{\mathrm{R}}\right)}{\partial \boldsymbol{\theta}_{\mathrm{R}}}\right|_{\mathrm{s} c}=c \boldsymbol{t}_{\mathrm{r}} \otimes \boldsymbol{t}_{\phi}
$$

\subsection{Cam joint}

The technique will be presented on a cam joint with a simple eccentric cam lobe profile. Setting the upper node of the rotating element $B$ as the slave node and the left-end node of the element $A$ as the master node (see Figure 4), the relation between the released translational displacement $\boldsymbol{r}_{\mathrm{R}}$ and the released rotation $\boldsymbol{\theta}_{\mathrm{R}}$ may be written as

$$
\boldsymbol{r}_{\mathrm{R}}=\boldsymbol{f}_{\mathrm{cam}}\left(\boldsymbol{\theta}_{\mathrm{R}}\right)=\left(R \cos \left|\boldsymbol{\theta}_{\mathrm{R}}\right|-R-a\right) \boldsymbol{t}_{\mathrm{r}}
$$

where $a$ and $2 R+a$ are the minimum and maximum released displacements of the arm $A$ and $\boldsymbol{t}_{\mathrm{r}}$ is the unit vector attached to the master node (direction in which the relative translation takes place).

The term $\partial \boldsymbol{f}\left(\boldsymbol{\theta}_{\mathrm{R}}\right) / \partial \boldsymbol{\theta}_{\mathrm{R}}$ for the cam joint is derived as

$$
\left.\frac{\partial \boldsymbol{f}\left(\boldsymbol{\theta}_{\mathrm{R}}\right)}{\partial \boldsymbol{\theta}_{\mathrm{R}}}\right|_{\mathrm{cam}}=-R \sin \left|\boldsymbol{\theta}_{\mathrm{R}}\right| \boldsymbol{t}_{\mathrm{r}} \otimes \boldsymbol{t}_{\phi}
$$

\section{ENERGY AND MOMENTUM CONSERVING ALGORITHM FOR BEAMS AND JOINTS}

The energy-momentum conserving algorithm presented in this section allows the interpolation of nodal unscaled rotations. This is in contrast to the energy and momentum conserving algorithm given in [14] and applied to systems with joints in Reference [3] which interpolates incremental tangent-scaled rotations. Interpolation of tangent-scaled rotations leads to a loss of strain-invariance [15]. The present formulation, which is an extension of [16] to systems with joints, restores this important property. 


\subsection{Energy and momentum conservation for $3 D$ beams}

The algorithm stems from the following momentum conserving weak form:

$$
\sum_{i=1}^{N} \boldsymbol{p}_{\mathrm{ts}}^{i} \cdot\left[\boldsymbol{q}_{k}^{i}\left(\mathbf{N}_{n+(1 / 2)}, \mathbf{M}_{n+(1 / 2)}\right)+\boldsymbol{q}_{d}^{i}\right]=0
$$

that uses the following definitions of the internal force vector $\boldsymbol{q}_{k}^{i}$ and the inertial force vector $\boldsymbol{q}_{d}^{i}$,

$$
\begin{aligned}
\mathbf{q}_{k}^{i}\left(\mathbf{N}_{n+(1 / 2)}, \mathbf{M}_{n+(1 / 2)}\right) & =\int_{L}\left[\begin{array}{cc}
I^{\prime i} \mathbf{I} & \mathbf{0} \\
-\hat{\boldsymbol{r}}_{n+(1 / 2)}^{\prime} I^{i} & I^{\prime i} \mathbf{I}
\end{array}\right]\left\{\begin{array}{c}
\boldsymbol{\Lambda}_{n+(1 / 2)} \mathbf{N}_{n+(1 / 2)} \\
\mathbf{S}(\boldsymbol{\alpha})^{-1} \boldsymbol{\Lambda}_{n} \mathbf{M}_{n+(1 / 2)}
\end{array}\right\} \mathrm{d} s \\
\mathbf{q}_{d}^{i} & =\frac{1}{\Delta t} \int_{L}\left\{\begin{array}{c}
A_{\rho}\left(\dot{\boldsymbol{r}}_{n+1}-\dot{\boldsymbol{r}}_{n}\right) I^{i} \\
\left(\boldsymbol{\pi}_{n+1}-\boldsymbol{\pi}_{n}\right) I^{i}
\end{array}\right\} \mathrm{d} s
\end{aligned}
$$

where $(\bullet)^{\prime}$ and $(\bullet)$ are the derivatives with respect to the arc-length parameter of the beam and time respectively, $\Delta t$ is the time-step size, $A_{\rho}=\rho A, A$ is the area of the cross section, $\boldsymbol{\pi}=\boldsymbol{\Lambda} \mathbf{J}_{\rho} \mathbf{W}$ is the specific angular momentum, $\mathbf{J}_{\rho}=\rho \operatorname{diag}\left(I_{2}+I_{3} I_{2} I_{3}\right)$ is the inertial tensor, $\mathbf{W}$ is the body attached angular velocity, $\rho$ is the density of the material, $I_{2}$ and $I_{3}$ are second moments of area of the cross section with respect to the principal axes of inertia, $(\bullet)_{n+\frac{1}{2}}=\frac{1}{2}\left((\bullet)_{n}+(\bullet)_{n+1}\right), \boldsymbol{r}$ is the position vector of the centroid axis, $\mathbf{N}$ is the stress resultant and $\mathbf{M}$ is the moment resultant in the cross section. Also, $\mathbf{S}(\boldsymbol{\alpha})^{-1}=1 /\left(1+(1 / 4)|\boldsymbol{\alpha}|^{2}\right)\left(\mathbf{I}+\frac{1}{2} \hat{\boldsymbol{\alpha}}\right)$ with $\boldsymbol{\alpha}=(\tan |\boldsymbol{\alpha} / 2| /|\boldsymbol{\alpha} / 2|) \omega$, and $I^{i}$ are the standard Lagrangian polynomials. Vector $\boldsymbol{p}_{\mathrm{ts}}^{i}$ has completely arbitrary nodal test parameters in the present momentum conserving algorithm.

Although various momentum conserving algorithms are possible, the form (17), taken here for further analysis, has been deduced in Reference [12] in such a way to keep the force vectors as close as possible to the energy conserving form given in Reference [14]. The internal and the inertial forces (17) do not provide a full energy conservation, but as described in Reference [16], this important property can be restored by adding an extra term into the weak form multiplied by a parameter $\beta$, to be found from the condition that the weak form is equal to the energy increment over a time-step:

$$
\sum_{i=1}^{N} \boldsymbol{p}_{\mathrm{ts}}^{i} \cdot\left[\boldsymbol{q}_{k}^{i}\left(\mathbf{N}_{n+(1 / 2)}, \mathbf{M}_{n+(1 / 2)}\right)+\boldsymbol{q}_{d}^{i}+\beta \boldsymbol{q}_{k}^{i}\left(\mathbf{N}_{d}, \mathbf{0}\right)\right]=H_{n+1}-H_{n}
$$

where $\mathbf{N}_{d}=\mathbf{N}_{n+1}-\mathbf{N}_{n}, H$ is the total energy, and a non-zero $\boldsymbol{p}_{\mathrm{ts}}^{i}$ can be arbitrarily chosen. In order to facilitate the application of the method to systems with joints, $\boldsymbol{p}_{\mathrm{ts}}^{i}$ will be chosen as

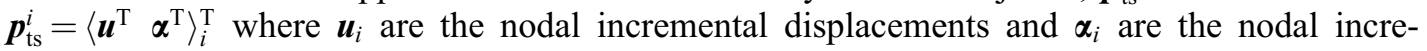
mental tangent-scaled rotations. Since the energy increment should be zero for a conservative problem (no external loads are considered here), the weak form in (18) leads to the following dynamic equilibrium equation:

$$
\boldsymbol{g}^{i}=\boldsymbol{q}_{k}^{i}\left(\mathbf{N}_{n+(1 / 2)}, \mathbf{M}_{n+(1 / 2)}\right)+\boldsymbol{q}_{d}^{i}+\beta \boldsymbol{q}_{k}^{i}\left(\mathbf{N}_{d}, \mathbf{0}\right)=\mathbf{0}
$$


and the value for $\beta$ follows as

$$
\beta=\frac{H_{n+1}-H_{n}-\sum_{i}^{N} \boldsymbol{p}_{\mathrm{ts}}^{i} \cdot\left[\boldsymbol{q}_{k}^{i}\left(\mathbf{N}_{n+(1 / 2)}, \mathbf{M}_{n+(1 / 2)}\right)+\boldsymbol{q}_{d}^{i}\right]}{\sum_{i}^{N} \boldsymbol{p}_{\mathrm{ts}}^{i} \cdot \boldsymbol{q}_{k}^{i}\left(\mathbf{N}_{d}, \mathbf{0}\right)}
$$

Full details on the implementation, including the linearisation of (18), are given in Reference [12].

\subsection{Application to systems with joints}

Extending the method to beam systems with joints can be done in a way similar to that of Section 3. We define the slave, master and released incremental displacements and tangentscaled rotations that will be written as $\boldsymbol{p}_{\mathrm{s}, \mathrm{ts}}, \boldsymbol{p}_{\mathrm{m}, \mathrm{ts}}$ and $\boldsymbol{p}_{\mathrm{R}, \mathrm{ts}}$ respectively. In addition, the vector composed of the released and master increments will be denoted as $\boldsymbol{p}_{\mathrm{Rm}, \mathrm{ts}}$. Following the notation used in Reference [3] the general relation between $\boldsymbol{p}_{\mathrm{s}, \mathrm{ts}}$ and $\boldsymbol{p}_{\mathrm{Rm}, \mathrm{ts}}$ is written as

$$
\boldsymbol{p}_{\mathrm{s}, \mathrm{ts}}=\tilde{\mathbf{N}} \boldsymbol{p}_{\mathrm{Rm}, \mathrm{ts}} \quad \text { with } \tilde{\mathbf{N}}=\left[\begin{array}{cccc}
\mathbf{C} & \mathbf{0} & \mathbf{I} & \mathbf{D} \\
\mathbf{0} & \mathbf{A} & \mathbf{0} & \mathbf{I}
\end{array}\right]
$$

It was proven in Reference [3] that this relationship serves to extend any existing conservation properties of an underlying beam formulation onto systems with joints (with independent degrees of freedom) for the matrices $\mathbf{A}, \mathbf{C}$ and $\mathbf{D}$ given as

$$
\begin{aligned}
& \mathbf{A}=\frac{1}{1-\frac{1}{4} \boldsymbol{\alpha}_{\mathrm{m}} \cdot \boldsymbol{\Lambda}_{\mathrm{m}, n} \boldsymbol{\alpha}_{\mathrm{R}}} \mathbf{S}^{\mathrm{T}}\left(\boldsymbol{\alpha}_{\mathrm{m}}\right) \boldsymbol{\Lambda}_{\mathrm{m}, n} \\
& \mathbf{C}=\left(\mathbf{I}-\frac{1}{4} \hat{\boldsymbol{\alpha}}_{\mathrm{m}}^{2}\right) \boldsymbol{\Lambda}_{\mathrm{m}, n+(1 / 2)} \\
& \mathbf{D}=-\frac{1}{2}\left(\widehat{\boldsymbol{\Lambda}}, n_{\boldsymbol{r}_{\mathrm{R}, n}}+{\widehat{\boldsymbol{\Lambda}_{\mathrm{m}, n+1} \boldsymbol{r}_{\mathrm{R}, n+1}}}\right)
\end{aligned}
$$

In contrast to Reference [3] where the energy and momentum conserving residual proposed in Reference [14] was taken for the underlying beam formulation, here we use residual (19) and apply it to joints with dependent degrees of freedom from Section 3. Assuming that the kinematic relation (9) can be expressed in an incremental form as

$$
\boldsymbol{u}_{\mathrm{R}}=\boldsymbol{r}_{\mathrm{R}, n+1}-\boldsymbol{r}_{\mathrm{R}, n}=\mathbf{G} \boldsymbol{\alpha}_{\mathrm{R}}
$$

a modification of $\tilde{\mathbf{N}}$ given in (21) is obtained as

$$
\underline{\tilde{\mathbf{N}}}=\left[\begin{array}{cccc}
\mathbf{0} & \mathbf{C G} & \mathbf{I} & \mathbf{D} \\
\mathbf{0} & \mathbf{A} & \mathbf{0} & \mathbf{I}
\end{array}\right]
$$

The energy-momentum conserving algorithm in (18) thus becomes

$$
H_{n+1}-H_{n}=\sum_{i}^{N} \boldsymbol{p}_{\mathrm{Rm}, \mathrm{ts}}^{i} \cdot \underline{\mathbf{N}}^{\mathrm{T}}\left[\boldsymbol{q}_{k}^{i}\left(\mathbf{N}_{n+(1 / 2)}, \mathbf{M}_{n+(1 / 2)}\right)+\boldsymbol{q}_{d}^{i}+\beta \boldsymbol{q}_{k}^{i}\left(\mathbf{N}_{d}, \mathbf{0}\right)\right]=0
$$


The kinematic relation for the screw joint and rack-and-pinion joint has been given in (12). The expression for $\boldsymbol{u}_{\mathrm{R}}$ is obtained from the difference between $\boldsymbol{r}_{\mathrm{R}, n+1}$ and $\boldsymbol{r}_{\mathrm{R}, n}$ as

$$
\boldsymbol{u}_{\mathrm{R}}=c \boldsymbol{t}_{\mathrm{r}} \otimes \boldsymbol{t}_{\phi}\left(\boldsymbol{\theta}_{\mathrm{R}, n+1}-\boldsymbol{\theta}_{\mathrm{R}, n}\right)=c \boldsymbol{t}_{\mathrm{r}} \otimes \boldsymbol{t}_{\phi} \boldsymbol{\omega}_{\mathrm{R}}=\frac{c \arctan \left|\boldsymbol{\alpha}_{\mathrm{R}} / 2\right|}{\left|\boldsymbol{\alpha}_{\mathrm{R}}\right| / 2} \boldsymbol{t}_{\mathrm{r}} \otimes \boldsymbol{t}_{\phi} \boldsymbol{\alpha}_{\mathrm{R}}
$$

i.e.

$$
\mathbf{G}=\frac{c \arctan \left|\boldsymbol{\alpha}_{\mathrm{R}} / 2\right|}{\left|\boldsymbol{\alpha}_{\mathrm{R}}\right| / 2} \boldsymbol{t}_{\mathrm{r}} \otimes \boldsymbol{t}_{\phi}
$$

where, for the screw-joint, $\boldsymbol{t}_{\mathrm{r}}=\boldsymbol{t}_{\phi}$.

Following a similar procedure as above, the increment of the released translations for the cam-lobe profile given in (14) reads

$$
\boldsymbol{u}_{\mathrm{R}}=R\left(\cos \left|\boldsymbol{\theta}_{\mathrm{R}, n+1}\right|-\cos \left|\boldsymbol{\theta}_{\mathrm{R}, n}\right|\right) \boldsymbol{t}_{\mathrm{r}}=R \frac{\cos \left|\boldsymbol{\theta}_{\mathrm{R}, n+1}\right|-\cos \left|\boldsymbol{\theta}_{\mathrm{R}, n}\right|}{\boldsymbol{t}_{\phi} \cdot \boldsymbol{\alpha}_{\mathrm{R}}} \boldsymbol{t}_{\mathrm{r}} \otimes \boldsymbol{t}_{\phi} \boldsymbol{\alpha}_{\mathrm{R}}
$$

thus giving

$$
\mathbf{G}=R \frac{\cos \left|\boldsymbol{\theta}_{\mathrm{R}, n+1}\right|-\cos \left|\boldsymbol{\theta}_{\mathrm{R}, n}\right|}{\boldsymbol{t}_{\phi} \cdot \boldsymbol{\alpha}_{\mathrm{R}}} \boldsymbol{t}_{\mathrm{r}} \otimes \boldsymbol{t}_{\phi}
$$

\section{NUMERICAL EXAMPLES}

\subsection{Freely rotating screw joint}

A vertical beam clamped at the bottom has a horizontal arm connected to it via a screw joint. The material properties for the two beams are shown in Figure 5. Both beams have been discretised using four linear elements. An initial distributed velocity in the $X$ direction and an angular velocity in the $Z$ direction that make the arm turn around and descend along the vertical beam are applied as depicted in Figure 5.

Three different time integration procedures with $\Delta t=0.02$ have been tested in this example: the Newmark method [17] with $\beta=\frac{1}{4}$ and $\gamma=\frac{1}{2}$ (trapezoidal rule), the momentum conserving algorithm similar to (17), but with $\boldsymbol{\Lambda}_{n+(1 / 2)}$ instead of $\mathbf{S}(\boldsymbol{\alpha})^{-1} \boldsymbol{\Lambda}_{n}$, and the energy-momentum algorithm given in (19) and (20). As shown in Figure 6 where the $Y$ displacement of the top of the vertical beam is plotted, the conserving algorithms give very similar results whereas the Newmark algorithm fails to converge due to energy blow-up at time $t=1.8653 \mathrm{~s}$ after a series of successive step halvings.

The analysis runs using the momentum conserving algorithm without any convergence problems, whereas in the energy-momentum conserving algorithm some time-step reductions have occasionally been necessary in order to achieve a converged solution. This fact reveals certain weaknesses in the method, which although energy and momentum conserving, is still not sufficiently robust. The momentum conserving algorithm is here capable of handling even larger time-steps. For this algorithm, however, a possibility of eventual energy blow-up must not be discounted [16]. 


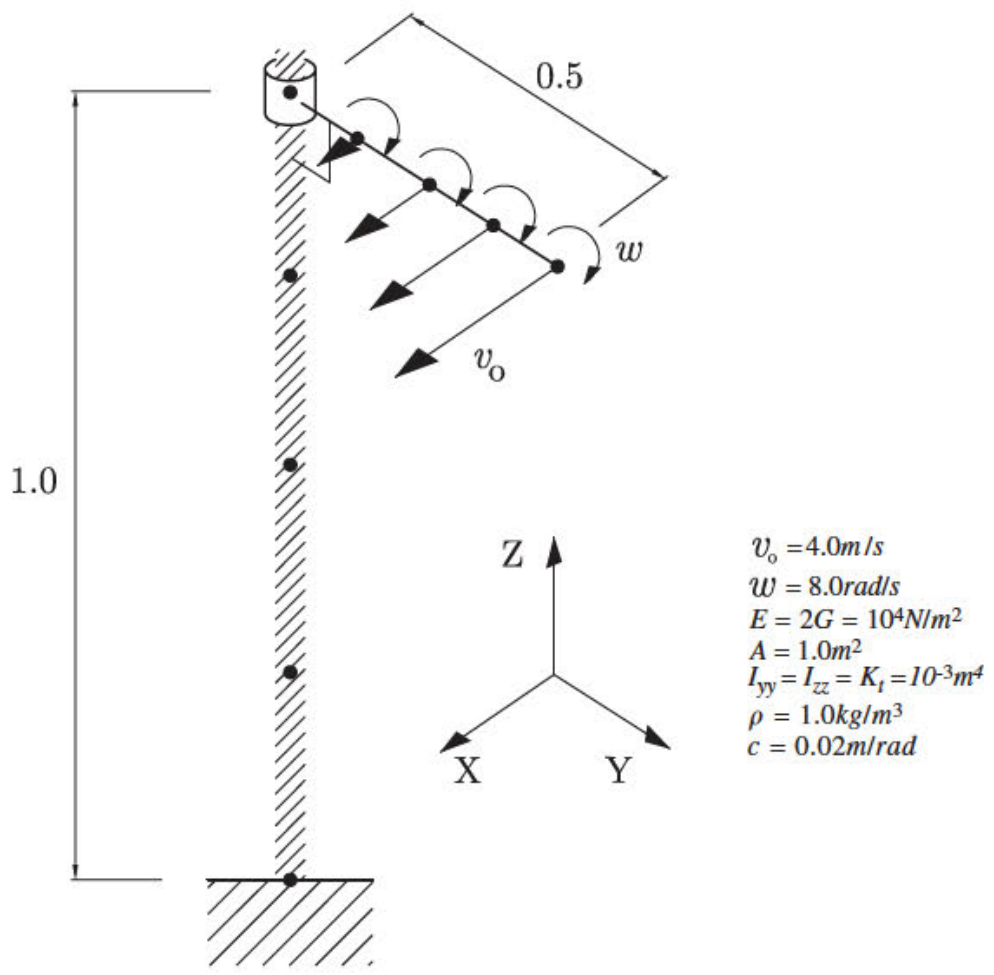

Figure 5. Turning arm with screw joint.

\subsection{Driven screw joint [10]}

In this example a vertical driver is attached to a fixed point $A$ through a revolute joint with its axis of rotation in the direction of $Z$ (perpendicular to the plane of the figure). The other end $B$ is connected to a horizontal beam first through a universal joint where the only constrained rotation is the one in the direction of the horizontal beam, and afterwards through a screw joint with the released rotation in the same direction. The pitch of the screw is $c=(2.4 / \pi / 3)=2.2918 \mathrm{~m} / \mathrm{rad}$ which corresponds to a twist of $60^{\circ}$ from point $R$ to point $T$. The beam is also physically twisted at the same ratio in such a way that at point $R$ the local axes $Y$ and $Z$ of the beam are rotated by $30^{\circ}$ with respect to the global axis $Y$ and $Z$ while at point $T$ they are rotated by $-30^{\circ}$ with respect to the same global axes. At point $T$ a rigid body $M$ is attached to the beam as depicted in Figure 7. The beam is attached to a fixed point $R$ by means of a universal joint that has the $X$ axis constrained. The geometrical and material properties of the beams are given in Figure 7.

The translation of the screw joint is prescribed during the analysis according to the function

$$
\boldsymbol{r}_{\mathrm{R}}=0.6(1-\cos 2 \pi t) \boldsymbol{t}_{\mathrm{r}}
$$

where $t$ is the time variable and $t_{\mathrm{r}}$ is the body-attached axis perpendicular to the cross section of the beam at point $B$ (initially in the direction of the global axis $X$ ). 


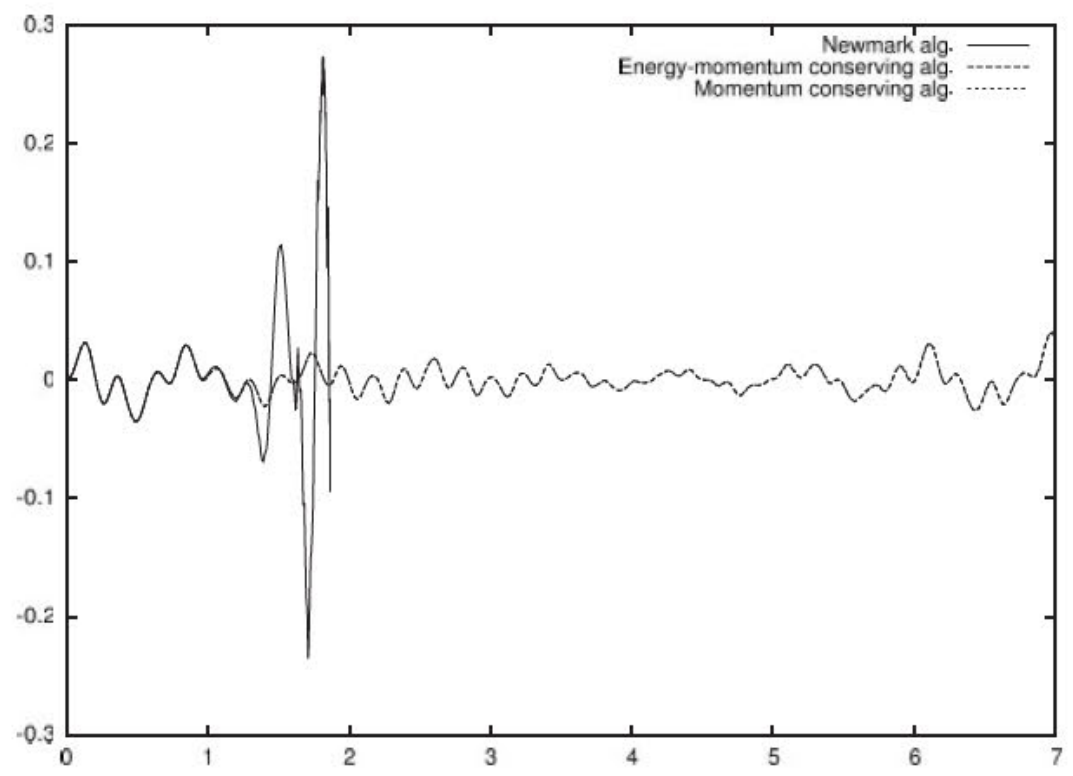

Figure 6. Displacement in the $Y$ direction of the top of the vertical beam for the freely rotating screw joint.

The driver and the beam have been discretised using 2 and 12 quadratic elements, respectively, and the total response time is $3 \mathrm{~s}$. The axial twist of the beam is modelled approximately by axially rotating each of the 12 untwisted elements with respect to each other in a way consistent with the geometry of the problem.

The problem has been run using the same three time-integration methods of the first example. For all the algorithms the time-step is set to $0.015 \mathrm{~s}$, and all of them successfully complete the analysis run for $3 \mathrm{~s}$. Newmark's method however has occasionally experienced convergence problems, which has necessitated time-step reductions at these time instants. Figures 8 and 9 show the out-of-plane displacement $u_{z}$ and rotation $\theta_{X}$ of the tip point $T$ for each time integration scheme. These plots agree very nicely with the original results [10] apart from some small differences during the last second of the simulation, which we believe are due to the different time integration scheme and the different spatial and time discretisation used.

\section{CONCLUSIONS}

The modifications added to the master-slave formulation in this work allow modelling a wider set of joints, some of them extensively used in flexible multibody systems. The general approach used in the present paper treats them from a single standpoint by adding small modifications to the standard master-slave approach.

The formulation has been implemented in a (statically) path-independent and invariant manner, and embedded within a dynamic time-stepping framework which enables using either the traditional end-point algorithms, or the conserving mid-point algorithms. 


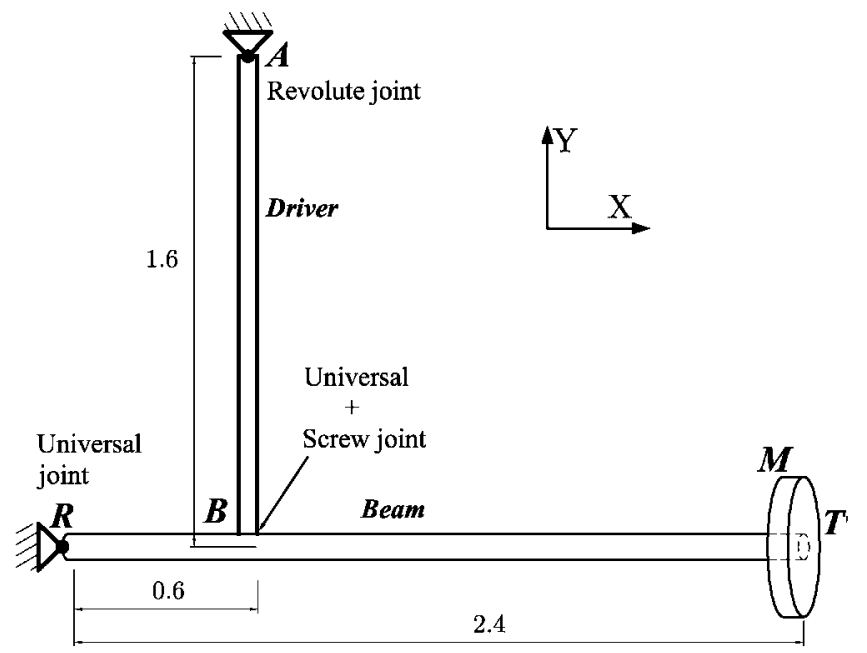

\begin{tabular}{|l|l|l|}
\hline Driver & Beam & Tip mass M \\
\hline$E A=44000 \mathrm{kN}$ & $E A=44000 \mathrm{kN}$ & $\mathrm{M}=40 \mathrm{~kg}$ \\
$E I_{y y}=23.0 \mathrm{kNm} \mathrm{m}^{2}$ & $E I_{y y}=300.0 \mathrm{kNm} \mathrm{m}^{2}$ & $\rho I_{y y}=0.225 \mathrm{kgm}^{2}$ \\
$E I_{z z}=300.0 \mathrm{kNm}^{2}$ & $E I_{z z}=23.0 \mathrm{kNm} \mathrm{m}^{2}$ & $\rho I_{z z}=0.225 \mathrm{kgm}^{2}$ \\
$G J=28.0 \mathrm{kNm}$ & $G J=28.0 \mathrm{kNm} m^{2}$ & \\
$G A_{y}=14000 \mathrm{kN}$ & $G A_{y y}=2800 \mathrm{kN}$ & \\
$G A_{z}=2800 \mathrm{kN}$ & $G A_{z}=14000 \mathrm{kN}$ & \\
$\rho I_{y y}=0.001 \mathrm{kgm}$ & $\rho I_{y y}=0.001 \mathrm{kgm}$ & \\
$\rho I_{z z}=0.011 \mathrm{kgm}$ & $\rho I_{z z}=0.011 \mathrm{kgm}$ & \\
\hline
\end{tabular}

Figure 7. Geometrical and material properties of the driven screw joint problem.

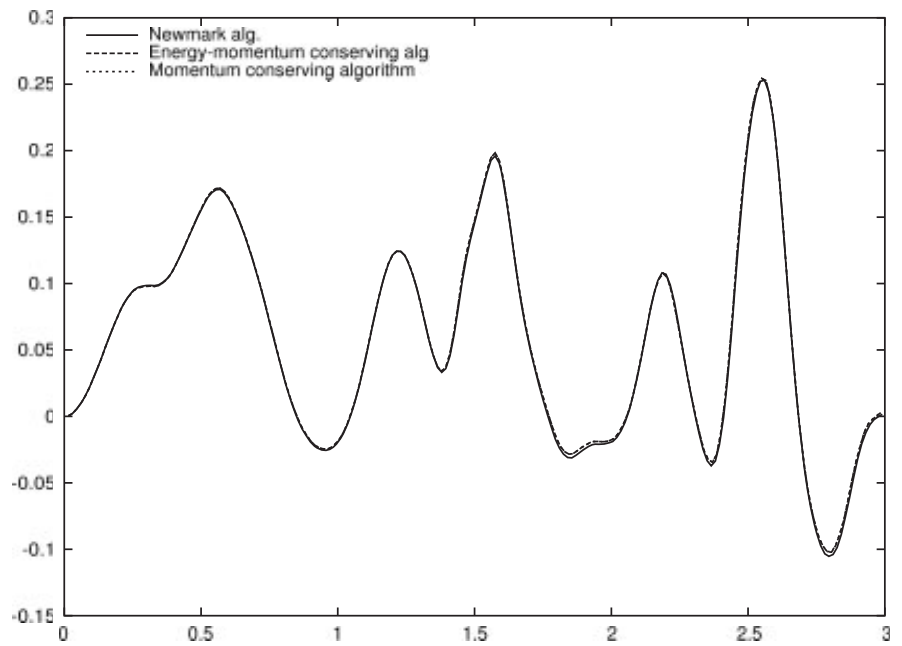

Figure 8. Out of plane displacement $u_{z}$ of the tip of the beam for the driven screw joint problem. 


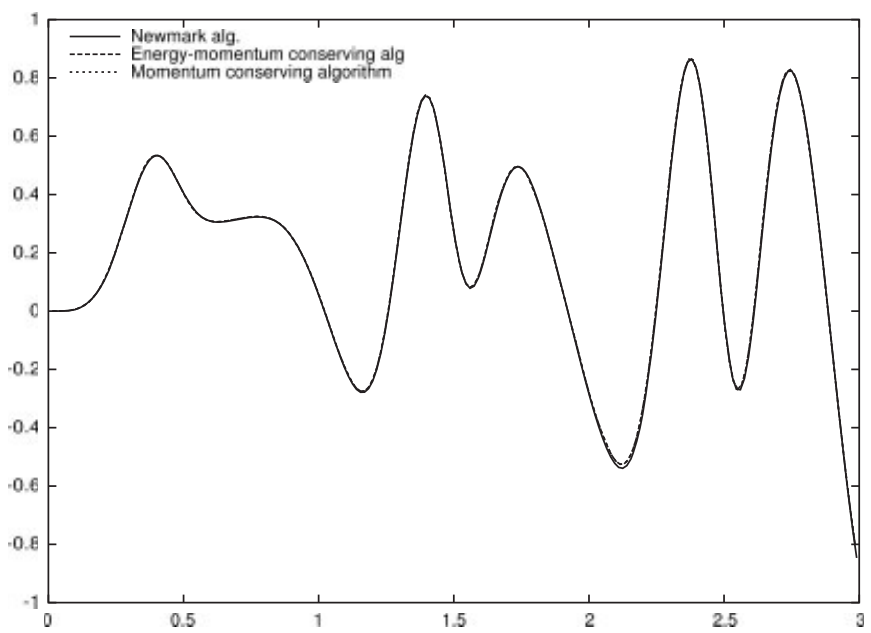

Figure 9. Rotation $\theta_{x}$ of the tip of the beam for the driven screw joint problem.

None of the advantages of the standard master-slave approach are spoilt, preserving the use of a minimum set of variables and exactly satisfying the constraint conditions of the joints.

\section{REFERENCES}

1. Crisfield MA, Jelenić G. Finite element analysis and deployable structures. In Deployable Structures: Theory and Applications. IUTAM: Cambridge, 6-9 September 1998; 87-96.

2. Jelenić G, Crisfield MA. Non-linear master-slave relationships for joints in 3D beams with large rotations. Computer Methods in Applied Mechanics and Engineering 1996; 135:211-228.

3. Jelenić G, Crisfield MA. Dynamics analysis of 3D beams with joints in presence of large rotations. Computer Methods in Applied Mechanics and Engineering 2001; 32-33:4195-4230.

4. Cardona A, Géradin MA. A beam finite element non-linear theory with finite rotations. International Journal for Numerical Methods in Engineering 1988; 26:2403-2438.

5. Ibrahimbegović A, Frey F, Kožar I. Computational aspects of vector-like parametrisation of three-dimensional finite rotations. International Journal for Numerical Methods in Engineering 1995; 38:3653-3673.

6. Jelenić G, Crisfield MA. Geometrically exact 3D beam theory: implementation of a strain-invariant finite element for statics and dynamics. Computer Methods in Applied Mechanics and Engineering 1999; 171:141-171.

7. Simo JC. A finite strain beam formulation. The three dimensional dynamics problem. Part I. Computer Methods in Applied Mechanics and Engineering 1985; 49:55-70.

8. Simo JC, Vu-Quoc L. On the dynamics in space of rods undergoing large motions - a geometrically exact approach. Computer Methods in Applied Mechanics and Engineering 1988; 53:125-161.

9. Ibrahimbegović A, Mamouri S. On rigid components and joint constraints in nonlinear dynamics of flexible multibody systems employing 3D geometrically exact beam model. Computer Methods in Applied Mechanics and Engineering 2000; 188:805-831.

10. Bauchau O, Bottasso CL. Contact conditions for cylindrical, prismatic, and screw Joints in flexible multibody systems. Multibody System Dynamics 2001; 5:251-278.

11. Cardona A, Géradin MA. Flexible Multibody Dynamics. A Finite Element Approach. John Wiley \& Sons: New York, 2001.

12. Muñoz J, Jelenić G, Crisfield M. Master-slave approach for the modelling of joints with dependent degrees of freedom in flexible mechanisms. Technical Report, Department of Aeronautics, Imperial College, London, August 2002.

13. Shigley JE, Uicker JJ. Theory of Machines and Mechanisms. (2nd edn). McGraw-Hill: New York, London, 1995. 


\section{J. MUÑOZ, G. JELENIĆ AND M. A. CRISFIELD}

14. Simo JC, Tarnow N, Doblare M. Non-linear dynamics of three-dimensional rods: exact energy and momentum conserving algorithms. International Journal for Numerical Methods in Engineering 1995; 38:1431-1473.

15. Jelenić G, Crisfield MA. Problems associated with the use of Cayley transform and tangent scaling for conserving energy and momenta in the Reissner-Simo beam theory. In press. Communications in Numerical Methods in Engineering.

16. Crisfield MA, Jelenić G. Energy/momentum conserving time integration procedures with finite elements and large rotations. In Computational Aspects of Nonlinear Structural Systems with Large Rigid Body Motion, Ambrósio JAC, Kleiber M (eds). IOS Press: Pultusk, Poland, 2-7 July 2000; 181-200.

17. Newmark NM. A method of computation for structural dynamics. Journal of Engineering Mechanics Division 1959; 85(EM3):67-94. 